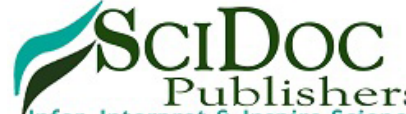 \\ Infer, Interpret \& Inspire Science
}

\author{
International Journal of Dentistry and Oral Science (IJDOS) \\ ISSN: 2377-8075
}

\section{Knowledge Of Antibiotic Prescription Habit Among Dental Students}

Research Article

Hanshika Ravi ${ }^{1}$, Dinesh Prabu ${ }^{2 *}$

${ }^{1}$ Saveetha Dental College and Hospitals, Saveetha Institute of Medical and Technical Sciences (SIMATS), Saveetha University, Chennai, India.

${ }^{2}$ Senior Lecturer, Department of Oral and Maxillofacial Surgery, Saveetha Dental College and Hospitals, Saveetha Institute of Medical and Technical Sciences (SIMATS), Saveetha University, Chennai, India.

\section{Abstract}

\begin{abstract}
Antibiotics are commonly prescribed by almost all dental practitioners. The prescription habits of dentists vary among different groups of practitioners which can lead to various complications. This study aims in assessing the knowledge and practice on antibiotic prescription among the undergraduate students of a private dental college in Chennai. This study was conducted as a questionnaire based online survey in a private dental institute in Chennai.The data collection was done through google forms. Data was analysed using SPSS software.A correlation test and chi square analysis was done. On chi square analysis, the results showed $\mathrm{p}$ value 0.692 . ( $>0.05)$, does not have significant association. Most of the dentists preferred amoxicillin as their choice of antibiotics (82.35\%). Majority of the dentists prescribed antibiotics for a period of 3-5 days (52.94\%) and they prescribed antibiotics based on the symptoms of the patients. Thus it can be concluded that the dentists had good knowledge on antibiotic prescription which is in contrary to many other studies, which may be attributed various limitations.
\end{abstract}

Keywords: Antibiotics; Dentists; Prescription Habits.

\section{Introduction}

Antibiotics and analgesics are the most commonly prescribed medicine by dental practitioners [17]. Antibiotics are chemical substances that are capable of destroying and inhibiting the growth of microorganisms such as bacteria and fungi [11]. Antibiotics have been used for many years to manage infections and are prescribed by general dental practitioners [19]. What does not kill you makes you stronger is probably the best for antibiotic resistant strains of bacteria developing because of unjustified antibiotic prescription [4]. Since their introduction, antibiotics have been successfully introduced in dental practices for management as well as prevention of infections [3]. Antibiotics may be prescribed in situations where dentists fail to give sufficient time for patient evaluation. They also antibiotics based on anecdotal experiences, heavy or just in case. Dentists prescribe antibiotics for orofacial pain, for localised signs and symptoms of infections without presence of systemic effects and clinical treatment may also be sufficient [4]. There are various dental procedures being done such as extractions, root canal therapy, prosthetic proce- dures etc.,[14]. Patients sometimes tend to self medicate due to counter availability of antibiotics. Unjust antibiotic use increases the incidence of antibiotic reactions that are allergic to patients [4].

Antibiotics resistance is a cause of major concern as more and more resistant strains are being seen [17]. There are many researchers that determine on how dentists prescribe antibiotics to patients and their knowledge on it.This study was undertaken to determine the antibiotic prescription habits and knowledge of dentists in a private dental college.

\section{Materials and Methods}

It is a questionnaire based cross sectional study conducted among the undergraduate students of a private dental college in Chennai. It is an online survey conducted to assess the knowledge on antibiotic prescription habits. Ethical board clearance was obtained.

The data collection was done through google forms. The questionnaire contained 20 questions about their knowledge, attitude

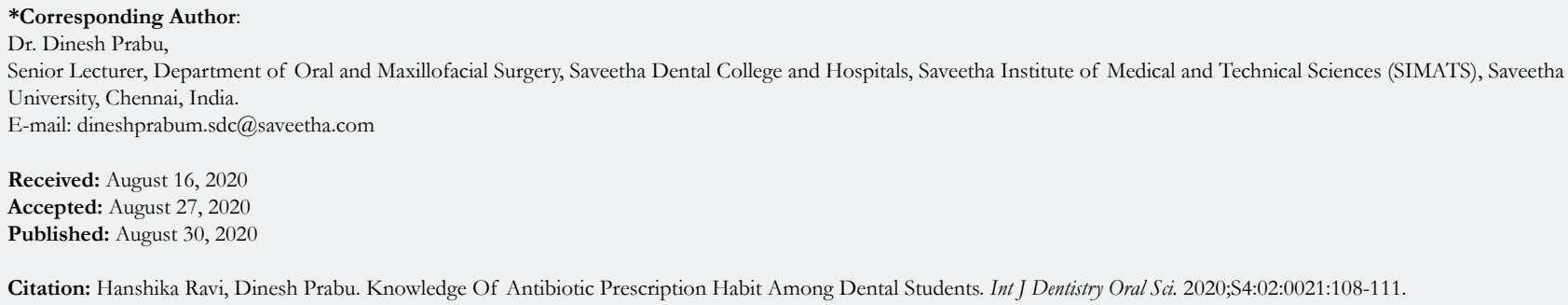

Copyright: Dinesh Prabu ${ }^{\circ}$ 2020. This is an open-access article distributed under the terms of the Creative Commons Attribution License, which permits unrestricted use, distribution and reproduction in any medium, provided the original author and source are credited. 
and practice of students in antibiotic prescription. Google forms were used to circulate the questionnaire. The data collected was tabulated in excel sheet.

The data collected was entered in excel sheet and output variable was defined in SPSS software. A correlation test and chi square analysis test was done. The independent variables were age and gender. The dependent variables were knowledge, attitude and practice of dentists in antibiotic prescription. The results were recorded and the difference was considered statistically significant.

\section{Results And Discussion}

From the analysis of the reports, all were undergraduate students, with two participants were in their third years, six people were final years and the remaining participants were interns. Most of the participants were undergraduate interns presumed to have a fair knowledge about their practice. In a similar study on biomedical waste management, intern students have the highest level of knowledge and practices toward dental waste disposal when compared to final year and 3rd year students. Hence, these findings imply that proper training, continuing education programs, and short-term courses are required to motivate the dental students and dental auxiliaries [15].

Penicillin and cephalexin were not in practice.In this sample, Amoxicillin was the most frequent first choice of antibiotics for dental problems followed by clindamycin and Amoxi-Clav. The results are in consistence with international preferences and standards [4]. The analgesic, anti-inflammatory, and antipyretic effects of NSAIDs are a result of the ability of these agents to inhibit cyclo-oxygenase (COX) enzymes, which catalyze the conversion of arachidonic acid to prostaglandins, which are fatty acids involved in the generation of pain, fever, and inflammation. [16]. A study was done to compare the effect of application of $0.2 \%$ chlorhexidine gel, a eugenol-based paste, together with a control group on the postoperative incidence of alveolar osteitis in patients having underwent extraction of third molars, recorded post op pain, inflammation, infection, and wound healing. This can be used as an alternative to antibiotics to relieve postoperative complication [5]. BTX-A was found very effective in the management of various facial pain conditions such as tension headache, migraine, myofascial pain, trigeminal neuralgia and in post-operative wound pain [15]. Relatively large number of participants prescribed antibiotics for a course of 3-5 days, followed by less than 3 days. Many dentists in private practice may prescribe antibiotics on demand of patients and to avoid complications [17]. Most of the dentists did not receive any emergency calls due to the antibiotic reactions $(88.2 \%)$, with a few people did receive it.(11.8\%). When asked about the ways to encounter this problem, most of the dentists asked their patients to stop the medication followed by an immediate medical consultation. Prescription of antihistamine was also chosen by some dentists to encounter it. Almost every student ps are aware of the pharmacokinetics and pharmacodynamics of the

Figure 1. Bar graph representing the correlation between the most commonly preferred by both the gender. $\mathrm{X}$ axis represents the most commonly preferred antibiotics and $\mathrm{Y}$ axis represents the number of patients.Among the male patients, $29.41 \%$ of the dentists preferred amoxicillin, $5.88 \%$ of them preferred amoxi clav. Among all the females $52.94 \%$ of the dentists preferred amoxicillin, $5.88 \%$ of the dentists preferred amoxi clav and $5.88 \%$ of the dentists preferred Clindamycin. On chi square analysis test results showed p value 0.692 . $(>0.05)$. Hence there was no significant association between gender and antibiotics.

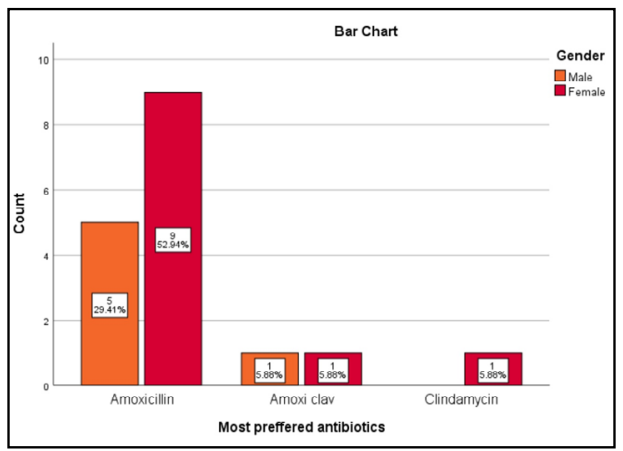

Figure 2. Bar chart representing the frequency association of most commonly preferred antibiotics by dentist post extractions. $\mathrm{X}$ axis represents the most preferred antibiotics and $\mathrm{Y}$ axis represents the number of respondents. Orange colour represents the dentists who prescribed amoxicillin, Green colour represents the number of dentists who prefer amoxi clav and blue colour represents the number of dentists who prefer clindamycin. $82.4 \%$ of the participants prescribe amoxicillin, $11.76 \%$ of the dentists prefer amoxi clav and $5.89 \%$ of the dentists prefer clindamycin. Amoxicillin is mostly preferred antibiotics by most of the dentists.

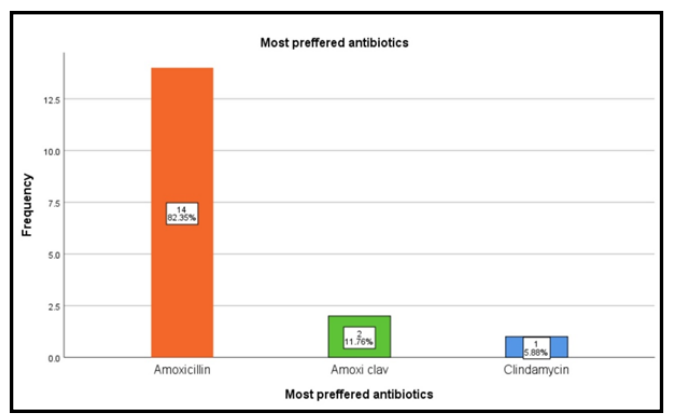


Figure 3. Bar chart representing the frequency distribution of duration of antibiotic course prescribed by dentists. $\mathrm{X}$ axis represents the duration of antibiotic course and $\mathrm{Y}$ axis represents the number of dentists. $47.06 \%$ of dentists prescribe antibiotics for less than three days (orange) and $52.94 \%$ of the dentists prescribe it for 3-5 days( Green). Majority of the dentists prescribed antibiotics for a period of 3-5 days.

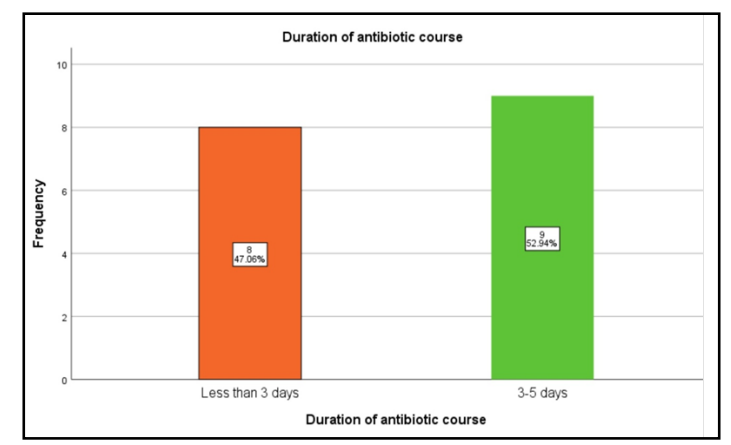

Figure 4. Bar graph representing the frequency distribution of dentists' opinion on prescription of antibiotics prior extractions. $\mathrm{X}$ axis represents the opinion of dentists and $\mathrm{Y}$ axis represents the number of dentists. $47.06 \%$ of the dentists accept antibiotic prescription before extraction is helpful for reducing pain and anxiety (Orange) whereas $52.94 \%$ of the dentists do not agree with it (Green ). Majority of the dentists do not accept antibiotics before extraction is necessary.

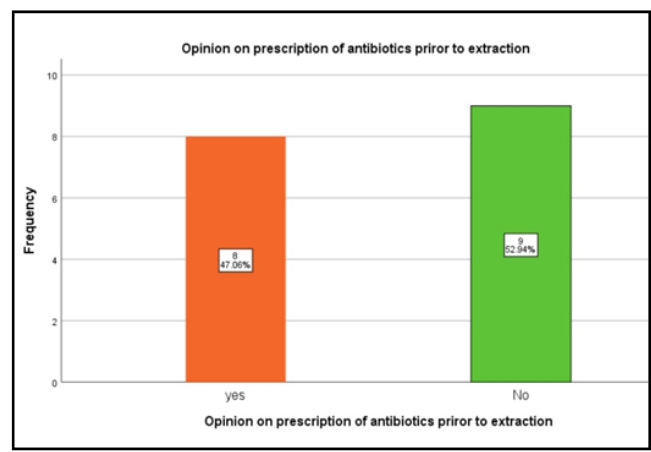

Figure 5. Bar graph representing the frequency distribution of most important factor for choosing a drug. $\mathrm{X}$ axis represents the factors for choosing a drug and $\mathrm{Y}$ axis represents the number of dentists. $\mathbf{7 0 . 5 9 \%}$ of the dentists prescribe antibiotics based on symptoms (orange) and $29.41 \%$ of the dentists prescribed antibiotics based on guidelines (Green). Majority of the dentists prescribe antibiotics based on patients 'symptoms.

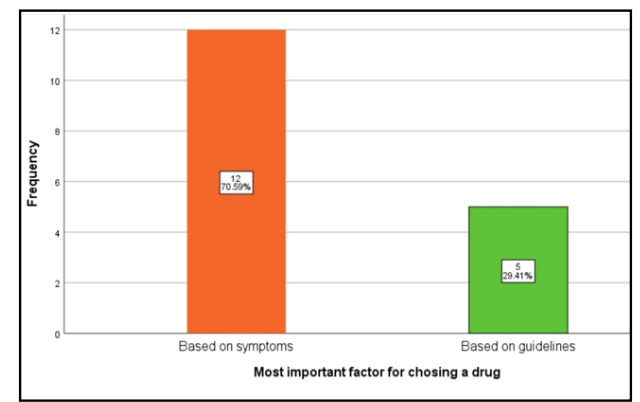

Figure 6. Bar chart representing the frequency distribution of dentists ' opinion on importance of medical history before starting any surgical procedure. $X$ axis represents the opinion of dentists and $Y$ axis represents the number of dentists. Every dentist accepted that the medical history of a patient is an important consideration before starting any surgical procedure (orange).

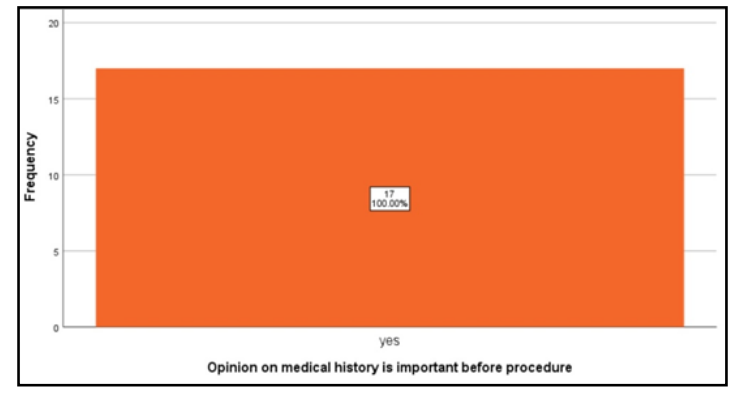


drugs that are prescribed. Also they are aware of the side effects of the drugs they prescribe. A few mentioned by them include nausea, vomiting, diarrhoea, gastritis, bloating, headache, rashes, stomach ache.

Everybody agrees that taking medical history prior to the procedure is important. For each patient, data on demographic information and details on type and site of injury, etiology of trauma, management undertaken, associated complications, alcohol use, the month during which the injuries occurred, and admissions should be collected [1]. Pharmacological methods like sedation can be used for reducing the pain and anxiety related to the treatment in indicated patients [8] for patients with dentophobias.

A similar study on Comparison of Pterygomaxillary Disjunction With Tuberosity Separation in Isolated Le Fort I Osteotomies was also done [2]. Knowledge of dental students about the newest guidelines for antibiotic prophylaxis for high-risk patients in dentistry and the correct application of these guidelines in different aspects are very important for a safe dental practice [6].

The steps taken to encounter a patient's antibiotic resistance includes giving test dosage, change of antibiotic, incomplete antibiotic course. Everyone are aware of the culture sensitivity test for antibiotics and its importance in prescribing antibiotics based on the nature of causative organisms. With improved survival rates due to the success of antiretroviral therapies, it is expected that more HIVpositive patients will require increasingly competent and compassionate health care, including oral care, in the near future [15]. Evaluation of Three-Dimensional Changes in Pharyngeal Airway Following Isolated Lefort One Osteotomy for the Correction of Vertical Maxillary Excess was done [18].

Yet a lot of oral and dental problems remain undiagnosed or untreated due to various reasons including alterations in gene expression. OSCC remains a major health problem and understanding the molecular basis of this malignancy is of great importance. Hardly, few studies have been carried out in the past to study the correlations of clinically relevant variables and the expression changes of possible oncogenic genes [7]. Other than medical management,with the advent of technology, newer non invasive medical management and use of lasers have gained acknowledgement in the management of other severe diseases [12]. A study was done to assess application of extended nasolabial flap versus buccal fat pad graft in the surgical management of oral submucous fibrosis [13]. From the above results it is said that the practitioners have a fair knowledge on prescription of antibiotics.

\section{Conclusion}

Within the limitations of the study, it can be concluded that the dentists had good knowledge on antibiotic prescription which is in contrary to many other studies. This difference may be due to various limitations such as single ethnic group, unicentric study and limited population.

\section{References}

[1]. Abhinav RP, Selvarasu K, Maheswari GU, Taltia AA. The Patterns and Etiology of Maxillofacial Trauma in South India. Ann Maxillofac Surg. 2019 Jan-Jun;9(1):114-117. Pubmed PMID: 31293938.

[2]. Christabel A, Anantanarayanan P, Subash P, Soh CL, Ramanathan M, Muthusekhar MR, et al. Comparison of pterygomaxillary dysjunction with tuberosity separation in isolated Le Fort I osteotomies: a prospective, multi-centre, triple-blind, randomized controlled trial. Int J Oral Maxillofac Surg. 2016 Feb;45(2):180-5. Pubmed PMID: 26338075.

[3]. Halboub E, Alzaili A, Quadri MF, Al-Haroni M, Al-Obaida MI, Al-Hebshi NN. Antibiotic Prescription Knowledge of Dentists in Kingdom of Saudi Arabia: An Online, Country-wide Survey. J Contemp Dent Pract. 2016 Mar 1;17(3):198-204. Pubmed PMID: 27207198.

[4]. Ismail F, Qazia S, Sajjada A. ANTIBIOTICS PRESCRIPTION HABITS AND KNOWLEDGE OF DENTISTS IN A LAHORE SAMPLE. Pakistan Oral \& Dental Journal. 2018 May 24;38(1):79-84.

[5]. Jesudasan JS, Wahab PU, Sekhar MR. Effectiveness of $0.2 \%$ chlorhexidine gel and a eugenol-based paste on postoperative alveolar osteitis in patients having third molars extracted: a randomised controlled clinical trial. $\mathrm{Br}$ J Oral Maxillofac Surg. 2015 Nov;53(9):826-30. Pubmed PMID: 26188932.

[6]. Kumar S, Sneha S. Knowledge and awareness regarding antibiotic prophylaxis for infective endocarditis among undergraduate dental students. Asian Journal of Pharmaceutical and Clinical Research. 2016;154.

[7]. Marimuthu M, Andiappan M, Wahab A, Muthusekhar MR, Balakrishnan A, Shanmugam S. Canonical Wnt pathway gene expression and their clinical correlation in oral squamous cell carcinoma. Indian J Dent Res. 2018 MayJun;29(3):291-297. Pubmed PMID: 29900911.

[8]. Kumar S. Relationship between dental anxiety and pain experience during dental extractions. Asian Journal of Pharmaceutical and Clinical Research. 2017;10(3):458.

[9]. Kumar S. The emerging role of botulinum toxin in the treatment of orofacial disorders: Literature update. Asian Journal of Pharmaceutical and Clinical Research. 2017;10(9):21-9.

[10]. Kumar S, Rahman RE. Knowledge, awareness, and practices regarding biomedical waste management among undergraduate dental students. Asian Journal of Pharmaceutical and Clinical Research. 2017;10(8):341.

[11]. Naveen N, Suhas PG, Vanishree N, Patnaik S, Bharath C, Prasad KS. Current trends in prescription of antibiotics among dentists working in various dental colleges of Bengaluru City, India a cross sectional study. Int J Oral Health Med Res. 2015;2(2):8-14.

[12]. Packiri S, Gurunathan D, Selvarasu K. Management of Paediatric Oral Ranula: A Systematic Review. J Clin Diagn Res. 2017 Sep;11(9):ZE06-ZE09. Pubmed PMID: 29207849.

[13]. Patil SB, Durairaj D, Suresh Kumar G, Karthikeyan D, Pradeep D. Comparison of Extended Nasolabial Flap Versus Buccal Fat Pad Graft in the Surgical Management of Oral Submucous Fibrosis: A Prospective Pilot Study. J Maxillofac Oral Surg. 2017 Sep;16(3):312-321. Pubmed PMID: 28717289.

[14]. Patturaja K, Pradeep D. Awareness of Basic Dental Procedure among General Population. Research Journal of Pharmacy and Technology. 2016 Sep $1 ; 9(9): 1349$.

[15]. Rahman RE, Mp SK. Knowledge, attitude, and awareness of dental undergraduate students regarding human immunodeficiency virus/acquired immunodeficiency syndrome patients. Asian J Pharm Clin Res [Internet]. 2017;10(5):175-80.

[16]. Rao TD, Kumar MS. Analgesic efficacy of paracetamol vs ketorolac after dental extractions. Research Journal of Pharmacy and Technology. 2018 Aug $1 ; 11(8): 3375-9$.

[17]. Tanwir F, Khan S. Antibiotic prescription habits of dentists in major cities of Pakistan. JPDA. 2011 Jul;20(03):160-63.

[18]. Vijayakumar Jain S, Muthusekhar MR, Baig MF, Senthilnathan P, Loganathan S, Abdul Wahab PU, et al. Evaluation of Three-Dimensional Changes in Pharyngeal Airway Following Isolated Lefort One Osteotomy for the Correction of Vertical Maxillary Excess: A Prospective Study. J Maxillofac Oral Surg. 2019 Mar;18(1):139-146. Pubmed PMID: 30728705.

[19]. Zahabiyoun S, Sahabi M, Kharazi MJ. Improving Knowledge of General Dental Practitioners on Antibiotic Prescribing by Raising Awareness of the Faculty of General Dental Practice (UK) Guidelines. J Dent (Tehran). 2015 Mar;12(3):171-6. Pubmed PMID: 26622268. 